\title{
Are Amphetamines Associated with Adverse Cardiovascular Events Among Elderly Individuals?
}

\author{
James R. Latronica, DO, Taylor J. Clegg, BS, Wen-Jan Tuan, DHA, MPH, MS, and \\ Curtis Bone, MD, MHS
}

Background: Prescription amphetamines are the most common pharmacological treatment for attention deficit hyperactivity disorder (ADHD) and use among older age groups is increasing. The purpose of this study is to assess the risk of adverse cardiovascular events among individuals older than 65 years.

Methods: We conducted a retrospective matched cohort study using TriNetX database with propensity score matching (PSM) to assess the odds of a cardiovascular event among individuals with ADHD exposed to amphetamine compared with individuals with ADHD who were not exposed to this medication. During the index period of January 1, 2018, through December 31, 2020, 13,233 individuals older than 65 years (mean age $=69$ years) met the study criteria.

Results: The cohort exposed to amphetamine had increased blood pressure and increased odds of cardiovascular events (odds ratio $[0 R], 6.16$; absolute risk difference $=3.31 \%$ ) compared with the control group.

Conclusions: Amphetamines have clear safety data in younger age cohorts; however, this safety data may not generalize to older populations. Additional research is warranted to clarify further exposure and subpopulation-level risk factors associated with adverse cardiovascular events among older patients. (J Am Board Fam Med 2021;34:1074-1081.)

Keywords: Amphetamine, Attention Deficit Disorder with Hyperactivity, Cardiology, Cardiovascular Diseases, Cohort Studies, Geriatric Psychiatry, Geriatrics, Heart Disease Risk Factors, Myocardial Infarction, Prescriptions, Retrospective Studies, Stroke

\section{Introduction}

Stimulants are a class of medication that is characterized by increased catecholamine levels and agonism of adrenergic receptors. ${ }^{1}$ They are noted to result in elevated energy levels, euphoria, libido, and enhanced cognition. ${ }^{2}$ Prescription stimulant use has accelerated in the United States in recent years with a nearly 6-

This article was externally peer reviewed.

Submitted 1 June 2021; revised 16 August 2021; accepted 19 August 2021.

From the University of Pittsburgh School of Medicine, Department of Psychiatry; Department of Family Medicine, Pittsburgh, PA (JRL); Penn State Health Milton S. Hershey Medical Center, Hershey, PA, (TJC, WJT); Penn State Health Milton S. Hershey Medical Center, Department of Family and Community Medicine, Hershey, PA (CB).

Funding: No funding was received for the execution or publishing of this study.

Conflict of interests: None of the four authors have any conflicts or competing interests to disclose.

Corresponding author: James R. Latronica, DO, University of Pittsburgh School of Medicine, 3501 Forbes Avenue, Oxford Building, Suite 860, Pittsburgh, PA 15213, Phone: (330) 416-4077 (E-mail: latronicajr3@upmc.edu). fold increase in prescriptions since $1993,{ }^{3}$ and a doubling from 2006 to $2016 .^{4}$ Although stimulants are utilized to address several conditions, ${ }^{1}$ the majority are prescribed for attention-deficit hyperactivity disorder (ADHD). ${ }^{5}$ ADHD is the most common behavioral disorder of childhood, and multiple studies have demonstrated stimulants are effective, safe, and well-tolerated in this age group. ${ }^{6-8}$ However, a recent study found that while prescriptions to individuals younger than 16years of age decreased between 2008 and 2018 , prescriptions to older adults increased by $355 \%$ during this time.?

Cardiovascular events among pediatric populations are considered rare beyond minor elevations in mean blood pressure and heart rate, ${ }^{10,11}$ but risks to adults are less clear. One study involving 806,182 person-years of observations among adults showed no increased risk of adverse cardiovascular events ${ }^{12}$; however, a separate study in adult populations showed an increased risk of transient ischemic attack (TIA) with ADHD medication. ${ }^{13}$ Additional studies 
focused on younger adults aged 18 to 44 years demonstrated increased risk of myocardial infarction ${ }^{14}$ and stroke $^{15}$ associated with amphetamine abuse. Stroke, myocardial infarction, and other adverse cardiovascular events such as arrhythmia and congestive heart failure may be mediated through hypertension, vasoconstriction, or platelet aggregation, which are all associated with amphetamine use. ${ }^{16}$

Although general cardiac physiology, traditional teaching, and Beers criteria suggest increased risk of amphetamine use in older adults, amphetamine prescriptions to older adults are growing. A recent systematic review focused on the utilization of stimulants in individuals 65 years of age and older concluded that they are clinically effective for multiple conditions, but more safety data are needed. ${ }^{13}$ This study aims to determine if amphetamine prescription among individuals aged 65 years and older is associated with adverse cardiovascular events.

\section{Methods}

The study applied a retrospective, longitudinal cohort design using electronic health records from 43 health care organizations on the research network of the TriNetX database in the United States (Cambridge, MA). TriNetX is a federated health research network that provides researchers access to de-identified, aggregated electronic health record data (demographics, diagnoses, procedures, medications, laboratory tests, and genomics) of more than 68 million patients from participating health care organizations and cancer registries.

No protected health information is made available in the TriNetX database. The platform is compliant with the security and confidentiality regulations of the Health Insurance Portability and Accountability Act of 1996. Because there was no patient-level identifiable data involved or accessed in the analysis, this research was determined to be exempt from the Institutional Review Board oversight.

The study population focused on older adults (age $\geq 65$ years) with established ADHD before age 65. To ensure care continuity, patients eligible for the analysis must have at least 1 or more clinical encounters in either ambulatory or inpatient settings during the index period from January 1, 2018, through December 31, 2020.

The intervention group of the study consisted of patients with a diagnosis of ADHD (ICD-10 code F90) prescribed amphetamine or its variants, including dextroamphetamine, methamphetamine, and lisdexamfetamine. Inclusion was restricted to a diagnosis of $\mathrm{ADHD}$ due to the high prevalence of amphetamine prescriptions among this population, as well as to mitigate the risk of unbalanced confounding variables. Patients with a diagnosis of ADHD that were not prescribed amphetamine medications in the index period were assigned to the control group. The analysis excluded individuals who had a history of adverse cardiovascular events before the index date for this study. The primary outcome of interest was the odds of adverse cardiovascular events. Adverse cardiovascular events were defined as occurrences composite of congestive heart failure, myocardial infarction, ischemic stroke, arrhythmia, and atrial fibrillation. The secondary outcome measures consisted of common laboratory metrics for assessing the clinical risk of atherosclerotic cardiovascular disease, including high-density lipoprotein (HDL) and low-density lipoprotein (LDL) cholesterol values, systolic blood pressure (SBP) and diastolic blood pressure (DBP), and hemoglobin A1c (A1c). Confounders between the intervention and control groups were considered, including patient characteristics (age, sex, and race/ethnicity) and comorbidities (body mass index, diabetes, essential hypertension, and hyperlipidemia).

We utilized a 1:1 propensity score matching (PSM) technique to balance the baseline characteristics between the 2 groups to reduce potential bias from confounders. We compared outcomes in patients with ADHD prescribed amphetamines and patients with ADHD who were not prescribed amphetamines using logistic regression modeling with control for demographics and comorbid conditions, based on patient cohorts before and after propensity score matching. All data queries and statistical analyses were performed on the TriNetX portal. We reported gender and ethnicity terms consistent with coding from TriNetX data capture. Detailed data information for diagnoses and laboratory tests is provided in Appendix 1.

\section{Results}

Study Population

A total of 13,233 patients with ADHD met the study selection criteria from 40 health care organizations. Of these patients, 4,966 individuals prescribed amphetamines were assigned to the 
Table 1. Demographic and Health Characteristics Before and After Propensity Score Matching

\begin{tabular}{|c|c|c|c|c|c|c|}
\hline \multirow[b]{2}{*}{ Characteristics } & \multicolumn{3}{|c|}{ Before propensity score matching } & \multicolumn{3}{|c|}{ After propensity score matching } \\
\hline & $\begin{array}{l}\text { Amphetamine } \\
(\mathrm{N}=4966)\end{array}$ & $\begin{array}{c}\text { Non- } \\
\text { Amphetamine } \\
(\mathrm{N}=8267)\end{array}$ & $P$ value & $\begin{array}{l}\text { Amphetamine } \\
(\mathrm{N}=4966)\end{array}$ & $\begin{array}{c}\text { Non- } \\
\text { Amphetamine } \\
(\mathrm{N}=4966)\end{array}$ & $P$ value \\
\hline Age, mean $\pm S D$ & $69.6 \pm 4.25$ & $70.1 \pm 4.77$ & $<.001$ & $69.6 \pm 4.25$ & $69.67 \pm 4.39$ & .42 \\
\hline Female, n (\%) & $3153(63.5)$ & $5191(62.8)$ & .42 & $3153(63.5)$ & $3158(63.6)$ & .69 \\
\hline \multicolumn{7}{|l|}{ Race/ethnicity, n (\%) } \\
\hline White & $4450(89.6)$ & $7351(88.9)$ & .22 & $4450(89.6)$ & $4512(90.9)$ & .04 \\
\hline Black & $110(2.2)$ & $251(3.0)$ & $<.01$ & $110(2.2)$ & $87(1.8)$ & .10 \\
\hline Hispanic/Latino & $70(1.4)$ & $159(1.9)$ & .03 & $70(1.4)$ & $64(1.3)$ & .60 \\
\hline Not Hispanic/Latino & $3927(79.1)$ & $6366(77.0)$ & $<.01$ & $3927(79.1)$ & $3960(79.7)$ & .41 \\
\hline Body mass index, mean $\pm \mathrm{SD}$ & $28.19 \pm 5.83$ & $28.61 \pm 6.08$ & $<.01$ & $28.19 \pm 5.83$ & $28.54 \pm 6.11$ & .06 \\
\hline Essential hypertension, n (\%) & $2523(50.8)$ & $4191(50.7)$ & .90 & $2523(50.8)$ & $2510(50.5)$ & .79 \\
\hline Diabetes, n (\%) & $741(14.9)$ & $1294(15.7)$ & .26 & $741(14.9)$ & $692(13.9)$ & .16 \\
\hline Hyperlipidemia, n (\%) & $2627(52.9)$ & $4547(55.0)$ & .02 & $2627(52.9)$ & $2590(52.2)$ & .46 \\
\hline
\end{tabular}

SD, standard deviation.

intervention group, while 8,267 individuals without an amphetamine prescription were attributed to the control group. The mean age and body mass index of the intervention group was slightly lower than those of the control group (Table 1). The intervention group had a higher proportion of people identified as "Female" and "White," while the control group had a greater percentage of people identified as "Black." The proportion of patients with "Hispanic/Latino" heritage was similar in both groups. A lower percent of individuals had hyperlipidemia in the intervention group. No significant difference in diabetes or essential hypertension was found between the 2 groups. After applying PSM, the study population included 4,966 patients prescribed amphetamines in the intervention group and 4,966 patients without amphetamine prescription in the control group. The groups were well balanced after applying PSM.

\section{Primary Outcome}

In the overall population, before PSM, the analysis identified 191 patients aged 65 years and older (3.85\%) with adverse cardiovascular events in the cohort prescribed amphetamines, compared with 45 patients older than 65 years $(0.54 \%)$ in the group without amphetamines (Table 2). In essence, the older adults prescribed amphetamines had 7.07 times the odds (confidence interval $[\mathrm{CI}]=5.12,9.76$ ) of developing adverse cardiovascular events than their counterparts who were not prescribed amphetamines. After
PSM, the odds of adverse cardiovascular events for older adults with amphetamine medications were 6.16 times the odds $(\mathrm{CI}=4.22,8.99)$ than those who did not take amphetamines. In terms of incidence, adverse cardiovascular events occurred at 38 per 1,000 in the experimental group, compared with 6 per 1,000 in the control group. Finally, myocardial infarction was a rare event in this cohort. This finding may be due to a relatively short follow-up period of 3 years. There was not a detectable difference in this outcome between exposed and unexposed patients.

\section{Secondary Outcome}

In this matched retrospective cohort study, patients older than 65 years on amphetamine therapy tended to show increased blood pressure and HDL, but there was not a detectable difference in LDL or A1c. Table 3 provides the list of key laboratory tests used to estimate a person's risk of heart disease or stroke following the Atherosclerotic Cardiovascular Disease (ASCVD) algorithm. ${ }^{17}$

\section{Discussion}

These data suggest that patients with a diagnosis of ADHD who are aged 65 years and older may be at elevated risk of a negative cardiovascular outcome if exposed to amphetamines. This risk may be mediated through known ASCVD metrics such as blood pressure; however, events may also be due to acute vasoconstriction and stress on a vulnerable cardiovascular system. Table 3 demonstrates that the mean SBP and 


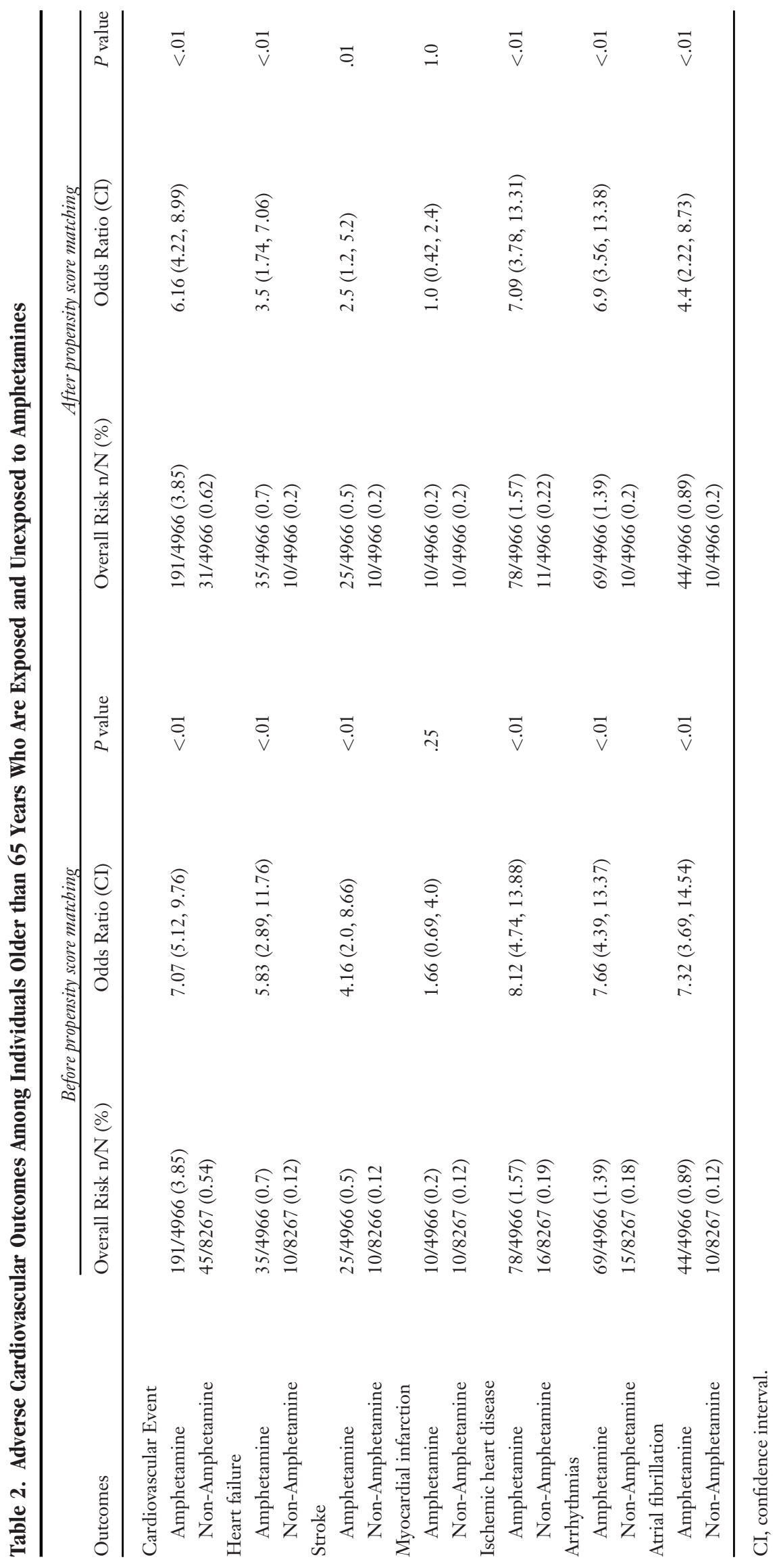


Table 3. Biological Metrics After Propensity Score Matching

\begin{tabular}{|c|c|c|c|c|}
\hline Biological Metric & Amphetamine (Mean \pm SD) & Non-Amphetamine (Mean \pm SD) & $\mathrm{t}$ Test & $P$ value \\
\hline HDL cholesterol (mg/dL) & $59.38 \pm 19.48$ & $57.62 \pm 18.22$ & 2.9 & $<.01$ \\
\hline LDL cholesterol (mg/dL) & $105.96 \pm 34.2$ & $106.57 \pm 34.52$ & -0.57 & .57 \\
\hline Diastolic blood pressure ( $\mathrm{mm} \mathrm{Hg}$ ) & $75.69 \pm 10.38$ & $74.63 \pm 10.54$ & 4.13 & $<.01$ \\
\hline Systolic blood pressure (mm Hg) & $129.99 \pm 17.24$ & $128.54 \pm 16.68$ & 3.3 & $<.01$ \\
\hline Hemoglobin A1c (\%) & $6.05 \pm 1.08$ & $6.02 \pm 1.06$ & 0.93 & .35 \\
\hline
\end{tabular}

HDL, high density lipoprotein cholesterol; LDL, low density lipoprotein cholesterol; SD, standard deviation.

HDL levels among individuals with amphetamine exposure were a fraction higher than those in the control group. On an individual level, these differences may not be clinically significant; however, this shows that population-level intermediate endpoints (HDL, LDL, A1c) are nearly equivalent (or favorable) for individuals with amphetamine exposure. Still, cardiovascular events are increased in this group. Further investigation regarding the relationship between intermediate endpoints (HDL, LDL, A1c, SBP, DBP) and cardiovascular events is warranted on an individual level.

Although previous studies have assessed cardiovascular risks associated with amphetamines and demonstrated increased heart rate and blood pressure in younger individuals, few studies have assessed risk specific to older populations. This is important to consider given the changes in cardiovascular physiology during the aging process. Most notably, as individuals age, they experience changes in myocardial elasticity and chemoelectrical conduction. In addition, elevated levels of circulating catecholamines and decreased $\beta$-adrenergic cellular receptor sensitivity are common. ${ }^{18}$ Atherosclerosis constitutes another significant age-related variation. ${ }^{19}$ All these age-related changes independently enhance the risk of adverse cardiovascular events, and it is plausible that the presence of amphetamines-which have innate characteristics that increase blood pressure and heart rate ${ }^{20}$ —creates complications for vulnerable cardiovascular systems in this age cohort.

A systematic review of cardiovascular outcomes related to amphetamines was published in 2012 and described studies among both adult and pediatric patients. ${ }^{21}$ They concluded that "stimulant use in children and adolescents likely did not result in negative cardiovascular outcomes, but found that there was a potential increased risk in adults." ${ }^{21}$ While this review surmised that there was no increased risk of negative cardiovascular outcomes in young and middle-aged adults (categorized as individuals between 25 and 64 years old), it did not involve data focused on individuals older than 65 years of age. Similarly, a retrospective study by Michielsen et al suggested that stimulants are a "safe treatment for older adults with $\mathrm{ADHD}$, under the condition that the cardiovascular parameters are monitored and appropriately managed during treatment." ${ }^{22}$ It is important to note that this was a single-site study in the Netherlands limited by sample size $(\mathrm{n}=113)$ and included patients aged 55 to 79 years. A small sample will bias results toward the null, and a heterogeneous population inclusive of people as young as 55 years may also mask the risk of amphetamines among older individuals. The data presented in our study represent a larger sample $(n=13,233)$ across 40 health care organizations and are exclusive to individuals older than 65 years. Given our data suggest significantly increased odds of negative cardiovascular outcomes, there is concern that smaller studies and those that do not adequately control for age may not detect significant cardiovascular risks among older individuals.

Even though our study focuses on a vulnerable population that has not been explored sufficiently, there are several important limitations to consider. First, the study was specific to patients with a diagnosis of ADHD. As such, the findings may not generalize to those treated with a stimulant for other conditions such as narcolepsy, depression, or dementia. Moreover, in this retrospective cohort, we cannot assess the level of amphetamine exposure, so we cannot comment on the influence of amphetamine dose or duration on cardiovascular outcomes. In addition, individuals in the control group who may have been exposed to amphetamines before the 3-year "washout" period could not be accounted for, which may create an underestimate of total 
amphetamine exposure. A prospective study or longitudinal data would better elucidate this relationship.

In addition, our study relied on the International Classification of Diseases, Version 10 (ICD-10) system for medical coding and billing. There are systemic challenges in establishing a diagnosis of $\mathrm{ADHD},{ }^{23}$ which may result in misclassification of both cases and controls in this study. Moreover, there are inherent errors in medical diagnosis codes that can lead to misclassification bias. These study data cannot be accessed by chart review to determine what level of misclassification may exist. Some ICD-10 diagnoses, such as "arrhythmia," may be vague or include benign conditions such as premature atrial contractions, which may not be clinically relevant indicators of risk.

Older individuals may be prescribed other, non-stimulant medication for ADHD, such as atypical antipsychotics. These may independently increase the risk of adverse cardiovascular events via their impact on cholesterol and QT intervals. We attempted to mitigate the risk of confounding by matching cohorts based on cholesterol levels and limiting the analysis to individuals with ADHD for both the exposed and control group. However, the potential impact of QT segment prolongation was not captured with these data. A recent study using the National Health and Nutrition Examination Survey dataset found that the use of antipsychotics for ADHD was too rare to include in their threshold for analysis ${ }^{24}$ (which suggests this variable is unlikely to influence the inferences of our study). However, we believe assessing the relationship between QT-prolonging medications, amphetamines, and cardiovascular events in older adults is worth evaluating.

Despite these limitations, we believe these data are worth considering when evaluating the risks and benefits of stimulant prescriptions in elderly patients, especially given that stimulants are categorically listed on the American Geriatrics Society's Beers Criteria as a medication class to "Avoid." 25 There is evidence they may offer therapeutic value for the treatment of depression, ${ }^{26-30}$ dementia ${ }^{31,32}$ and ADHD, ${ }^{33,34}$; however, benefits should always be considered in the context of potential risks. This study raises concerns that the geriatric population may have a different risk profile than adolescents and young adults treated with amphetamines.

\section{Conclusions}

This study demonstrates increased odds of negative cardiovascular events among older individuals with ADHD treated with amphetamines. Amphetamines have been studied extensively in younger age cohorts; however, this safety data may not generalize to older populations. Although the relationship between amphetamines and stroke, myocardial infarction, arrhythmia, and congestive heart failure has apparent biological plausibility, additional research is warranted to clarify exposure and subpopulation-level risk factors associated with adverse cardiovascular events among older patients. We also recognize that prescriptions for amphetamines may not be exclusive to a diagnosis of ADHD in older individuals. To minimize confounding variables in this retrospective study, we used a "single-diagnosis" population as a model to assess the relationship between amphetamines and cardiovascular events. The data from individuals with ADHD may not generalize to people with conditions such as depression and narcolepsy. Further clarity regarding potential risks of amphetamines in these subpopulations is critical to more fully inform patient-provider decisions related to stimulant use.

To see this article online, please go to: http://jabfm.org/content/ 34/6/1074.full.

\section{References}

1. Farzam K, Faizy RM, Saadabadi A. Stimulants. In: StatPearls. StatPearls Publishing; 2021. Updated August 6, 2021. Available at: http://www.ncbi.nlm. nih.gov/books/NBK539896/. Accessed March 9, 2021.

2. Calipari ES, Ferris MJ. Amphetamine mechanisms and actions at the dopamine terminal revisited. J Neurosci 2013;33:8923-5.

3. Safer DJ. Recent trends in stimulant usage. J Atten Disord 2016;20:471-7.

4. Piper BJ, Ogden CL, Simoyan OM, et al. Trends in use of prescription stimulants in the United States and Territories, 2006 to 2016. PLOS ONE 2018;13: e0206100.

5. Berman SM, Kuczenski R, McCracken JT, London ED. Potential adverse effects of amphetamine treatment on brain and behavior: a review. Mol Psychiatry 2009;14:123-42.

6. Rajeh A, Amanullah S, Shivakumar K, Cole J. Interventions in ADHD: a comparative review of stimulant medications and behavioral therapies. Asian J Psychiatr 2017;25:131-5. 
7. Arnsten AFT. Stimulants: therapeutic actions in ADHD. Neuropsychopharmacology 2006;31:2376-83.

8. Hechtman L, Abikoff H, Klein RG, et al. Academic achievement and emotional status of children with ADHD treated with long-term methylphenidate and multimodal psychosocial treatment. J Am Acad Child Adolesc Psychiatry 2004;43:812-9.

9. Grimmsmann T, Himmel W. The 10 -year trend in drug prescriptions for attention-deficit/hyperactivity disorder (ADHD) in Germany. Eur J Clin Pharmacol 2021;77:107-15.

10. Dalsgaard S, Kvist AP, Leckman JF, Nielsen HS, Simonsen M. Cardiovascular safety of stimulants in children with attention-deficit/hyperactivity disorder: a nationwide prospective cohort study. J Child Adolesc Psychopharmacol 2014;24:302-10.

11. Hammerness PG, Karampahtsis C, Babalola R, Alexander ME. Attention-deficit/hyperactivity disorder treatment: what are the long-term cardiovascular risks? Expert Opin Drug Saf 2015;14:543-51.

12. Habel LA, Cooper WO, Sox CM, et al. ADHD medications and risk of serious cardiovascular events in young and middle-aged adults. JAMA 2011;306: 2673-83.

13. Holick CN, Turnbull BR, Jones ME, Chaudhry S, Bangs ME, Seeger JD. Atomoxetine and cerebrovascular outcomes in adults. J Clin Psychopharmacol 2009;29:453-60.

14. Westover AN, Nakonezny PA, Haley RW. Acute myocardial infarction in young adults who abuse amphetamines. Drug Alcohol Depend 2008;96: 49-56.

15. Westover AN, McBride S, Haley RW. Stroke in young adults who abuse amphetamines or cocaine: a population-based study of hospitalized patients. Arch Gen Psychiatry 2007;64:495-502.

16. Diaconu CC, Dediu GN, Iancu MA. Drug-induced arterial hypertension - a frequently ignored cause of secondary hypertension: a review. Acta Cardiologica 2018;73:511-7.

17. Stone NJ, Robinson JG, Lichtenstein AH, American College of Cardiology/American Heart Association Task Force on Practice Guidelines, , et al. 2013 ACC/AHA guideline on the treatment of blood cholesterol to reduce atherosclerotic cardiovascular risk in adults. J Am Coll Cardiol 2014;63:2889-934.

18. Cheitlin MD. Cardiovascular physiology-changes with aging. Am J Geriatr Cardiol 2003;12:9-13.

19. Palombo C, Kozakova M. Arterial stiffness, atherosclerosis and cardiovascular risk: Pathophysiologic mechanisms and emerging clinical indications. Vascul Pharmacol 2016;77:1-7.

20. Stiefel G, Besag FMC. Cardiovascular effects of methylphenidate, amphetamines and atomoxetine in the treatment of attention-deficit hyperactivity disorder. Drug Saf 2010;33:821-42.

21. Westover AN, Halm EA. Do prescription stimulants increase the risk of adverse cardiovascular events?: A systematic review. BMC Cardiovasc Disord 2012;12:41.

22. Michielsen M, Kleef D, Bijlenga D, et al. Response and side effects using stimulant medication in older adults with ADHD: an observational archive study. J Atten Disord 2021; 1712-91.

23. Kalbag AS, Levin FR. Adult ADHD and substance abuse: diagnostic and treatment issues. Subst Use Misuse 2005;40:1955-81.

24. Dennis JA, Gittner LS, Payne JD, Nugent K. Characteristics of U.S. adults taking prescription antipsychotic medications, National Health and Nutrition Examination Survey 2013-2018. BMC Psychiatry 2020;20:483.

25. American Geriatrics Society 2015 updated Beers Criteria for potentially inappropriate medication use in older adults. J Amer Geri Soc 2015;63: 2227-46.

26. Roose SP, Schatzberg AF. The efficacy of antidepressants in the treatment of late-life depression. J Clin Psychopharmacol 2005;25:S1-7.

27. Alexopoulos GS, Katz IR, Reynolds CF, Carpenter D, Docherty JP, Expert Consensus Panel for Pharmacotherapy of Depressive Disorders in Older Patients. The expert consensus guideline series. Pharmacotherapy of depressive disorders in older patients. Postgrad Med 2001;1-86.

28. Satel SL, Nelson JC. Stimulants in the treatment of depression: a critical overview. J Clin Psychiatry 1989;50:241-9.

29. Lavretsky H, Reinlieb M, St Cyr N, Siddarth P, Ercoli LM, Senturk D. Citalopram, methylphenidate, or their combination in geriatric depression: a randomized, double-blind, placebo-controlled trial. Am J Psychiatry 2015;172:561-9.

30. Alexopoulos GS. Pharmacotherapy for Late-Life Depression. J Clin Psychiatry 2011;72:e4.

31. Dolder CR, Nicole Davis L, McKinsey J. Use of Psychostimulants in Patients with Dementia. Ann Pharmacother 2010;44:1624-32.

32. Young JJ, Lavakumar M, Tampi D, Balachandran S, Tampi RR. Frontotemporal dementia: latest evidence and clinical implications. Ther Adv Psychopharmacol 2018;8:33-48.

33. Sassi KLM, Rocha NP, Colpo GD, John V, Teixeira AL. Amphetamine use in the elderly: a systematic review of the literature. Curr Neuropharmacol 2020;18: 126-35.

34. Torgersen T, Gjervan B, Lensing MB, Rasmussen K. Optimal management of ADHD in older adults. Neuropsychiatr Dis Treat 2016;12: 


\begin{tabular}{|c|c|c|}
\hline Coding System & Code & Description \\
\hline \multicolumn{3}{|c|}{ Adverse cardiovascular event codes } \\
\hline ICD-10 & F90 & $\mathrm{ADHD}$ \\
\hline ICD-10 & I 48 & Atrial fibrillation \\
\hline ICD-10 & I49 & Arrhythmia \\
\hline ICD-10 & I50 & Congestive heart failure \\
\hline ICD-10 & I20; I22-I25 & Ischemic heart disease \\
\hline ICD-10 & $\mathrm{I} 21$ & Myocardial infarction \\
\hline ICD-10 & I63 & Stroke \\
\hline \multicolumn{3}{|c|}{ Amphetamine-related medication codes } \\
\hline RxNorm & 725 & Amphetamine \\
\hline RxNorm & 3288 & Dextroamphetamine \\
\hline RxNorm & 6816 & Methamphetamine \\
\hline RxNorm & 6901 & Methylphenidate \\
\hline RxNorm & 700810 & Lisdexamfetamine \\
\hline \multicolumn{3}{|c|}{ Comorbidity diagnosis codes } \\
\hline ICD-10 & E08-E13 & Diabetes (type I and type II) \\
\hline ICD-10 & $\mathrm{I} 10$ & Essential hypertension \\
\hline \multicolumn{3}{|c|}{ Diagnostic testing codes } \\
\hline LOINC & 9000 & Total cholesterol \\
\hline LOINC & 9001 & HDL \\
\hline LOINC & 9002 & LDL \\
\hline LOINC & 9037 & Hemoglobin A1c \\
\hline LOINC & 9083 & Body mass index \\
\hline LOINC & 9085 & Systolic blood pressure \\
\hline LOINC & 9086 & Diastolic blood pressure \\
\hline
\end{tabular}

ADHD, attention-deficient/hyperactivity disorder. HDL, high density lipoprotein cholesterol; ICD-10, International Classification of Diseases, Version 10; LDL, low density lipoprotein Cholesterol; LOINC, Logical Observation Identifiers Names and Codes. 